51 were assessed as being chemically compatible after 48 hours at ambient temperatures $\left(20-26^{\circ} \mathrm{C}\right)$. Midazolam appears to be drug at greatest risk of clinically significant chemical degradation. Microbiological stability was only reported for one combination.

Conclusions There is currently limited evidence for the physical, chemical and microbiological stability of solutions for continuous subcutaneous infusion over a period of 48 hours. More stability data is required before the use of 48 hour CSCIs can be evaluated for use within clinical practice.

Acknowledgements This project was funded through a grant from NHS Liverpool CCG.

\section{P-27 THE FREQUENCY AT WHICH DOSES AND DRUGS ADMINISTERED BY CSCIS ARE CHANGED: A SERVICE EVALUATION OF CLINICAL PRACTICE IN THE UNITED KINGDOM}

\begin{abstract}
1,2 James Baker, ${ }^{2,3}$ Andrew Dickman, ${ }^{2}$ Stephen Mason, ${ }^{1,2}$ John Ellershaw, ${ }^{1}$ Paul Skipper, ${ }^{4}$ Jenny Schneider. 'Royal Liverpool and Broadgreen University Hospitals NHS Trust, Liverpool, UK; ${ }^{2}$ Marie Curie Palliative Care Institute, University of Liverpool, Liverpool, UK; ${ }^{3}$ Blackpool Teaching Hospitals NHS Foundation Trust, Blackpool, UK; ${ }^{4}$ School of Biomedical Sciences and Pharmacy, The University of Newcastle, Newcastle, Australia
\end{abstract}

\subsection{6/bmjspcare-2017-00133.27}

Background Continuous subcutaneous infusions (CSCIs) are an effective method of multiple drug administration in end of life care when the oral route is compromised. ${ }^{1,2}$ At present, currently available chemical and microbiological stability data limits the infusion time of a CSCI to a maximum of 24 hours.

The ability to deliver prescribed medication by a continuous subcutaneous infusion (CSCI) over 48 hours may have numerous benefits in both patient care and health service resource utilisation.

Aim To gather data regarding the most frequently prescribed CSCI drug combinations and the frequency at which CSCI prescriptions are altered.

Design Prescription details of CSCIs containing a minimum of two drugs were collected by hospital pharmacists or members of palliative care teams at 10 Acute NHS Trusts on a daily basis for a minimum of 2 days, to a maximum of 7 days.

Setting/participants Anonymised CSCI prescription data were collected from an average of 50 patients at 10 Acute NHS Trusts in the United Kingdom.

Results and Conclusion Data collection is due for completion January 2017 and results will be presented.

Acknowledgements This project was funded through a grant issued by NHS Liverpool CCG.

\section{REFERENCES}

1. Ellershaw J, Neuberger RJ, Ward C. Care of the dying patient: the last hours or days of life. BMJ 2003;326:30-4.

2. Dickman A, Scott J. Evaluating the frequency of medication adjustments to continuous subcutaneous infusions in palliative care: Is there evidence to support 48hourly infusions? In Multinational Association of Supportive Care in Cancer (MASCC). Copenhagen, 2015.

\section{P-28 STAFF EXPERIENCES OF DELIRIUM IN THE HOSPICE SETTING}

Kerry Waterfield, Rachel Kiltie, Jonathan Pickard, Ulka Karandikhar, Fiona MacCormick, Donna Weiand, Felicity Dewhurst, Jen Vidrine, Grace Rowley, Paul Coulter. Health Education North East, Newcastle, UK

10.1136/bmjspcare-2017-00133.28

Background Delirium affects up to $88 \%$ of patients with advanced cancer and is associated with significant morbidity and mortality. Despite this it remains under recognised in hospice settings and the management is not always in line with national guidelines. This research study explores the views of staff nurses and healthcare assistants in hospices in order to identify underlying reasons for this.

Method Semi-structured qualitative interviews were conducted with registered nurses (12) and healthcare assistants (6) in three North-East England hospices. Data was analysed using interpretative phenomenological analysis.

Results The main themes to emerge were knowledge and management. Data highlight that knowledge of delirium is variable leading to uncertainty about what constitutes delirium in hospice inpatients with subsequent difficulties in management.

Subthemes in management include the emotional response evoked by caring for patients with delirium, ensuring patient safety within the hospice environment and staff perceptions about the appropriate place of care. Disparities in staff opinion are apparent between drug and non-drug approaches and there is also concern about the possible effects of medication.

Conclusion Results from three different hospices across the North East region consistently highlight similar barriers to the assessment and management of delirium. The data provides valuable insights which help to guide future staff education and multidisciplinary team working in order to improve the care of patients with delirium.

\section{P-29 STRIVING TO REMAIN RESPONSIVE: A RETROSPECTIVE ANALYSIS OF LONGER LENGTHS OF STAYON AN INPATIENT PALLIATIVE CARE UNIT}

Dominique Wakefield, Patrick Smith, Helen McGee. Hospice In The Weald, Tunbridge Wells, UK

10.1136/bmjspcare-2017-00133.29

Background Current UK agendas for end of life care advocate 'timely specialist palliative care' and 'effective' hospices that are 'responsive to people's needs'. Hospices need to maintain capacity for admissions, whilst facing increasing demands from a rapidly growing, multimorbid, complex population. Hospices cannot afford to accommodate protracted lengths of stay. We aimed to identify patient and service factors associated with hospice in-patient longer lengths of stay (LLOS), 21 days to enhance our ability to provide quality, effective and equitable care.

Methods Mixed methods: Retrospective cohort review of all LLOS admissions in 2015, analysing sociodemographic and disease variables contrasted against a retrospective case-control analysis of admissions totalling 7-20 days. This was 
complemented by a retrospective case-control, thematic analysis of electronic patient records, to examine the patient journey throughout the duration of the hospice stay.

Results LLOS admissions accounted for 23\%(76) of admissions to our hospice in 2015 (2342 bed days), median stay 28 days, 55\% female, median age 69 years, 96\% (73 admissions) referred for symptom control. Admission outcome: 38\% (29) of patients discharged, of which 59\% (17) had a new care arrangement following their stay.

No significant findings when analysing the following against LLOS admissions and admission outcome: Patient age, gender, primary diagnosis, number of comorbidities, presence of formal social care support, and permanent residence.

Thematic analysis identified 4 overarching themes implicated in LLOS: Uncertainty, In-house interventions, clinical and social complexity. This analysis emphasised, in particular, the interplay between communicating and managing uncertainty and social complexity.

Conclusion Our results would suggest that there are no sociodemographic or disease factors associated with LLOS. Thematic analysis provides an alternative and successful method of service evaluation. Thematic results emphasise the need for research into managing complexity and uncertainty in addition to highlighting the fact that our growing capacity to provide in-house interventions comes, ultimately, at a cost to bed availability.

\section{P-30 IMPROVING COMMUNICATION FROM ONCOLOGY TO PRIMARY CARE IN A LARGE CANCER CENTRE}

${ }^{1,2}$ Annette Edwards, ${ }^{1}$ Paul Hatfield, ${ }^{1}$ Fiona Hicks. 'Leeds Teaching Hospitals NHS Trust, Leeds, UK; ${ }^{2}$ Sue Ryder Wheatfields Hospice, Leeds, UK

\subsection{6/bmjspcare-2017-00133.30}

Background Recognising that people are entering the last phase of illness isn't always straightforward, and having conversations about treatment aims and planning for future care is not easy. In light of this a Senior Clinician Development Programme was established, comprising 7 consultants in different specialties and a GP to give a community perspective, facilitated by 2 palliative medicine consultants. This ran for 18 months from October 2011.

The following audit specifically looked at oncology, assessing if there was improvement in communication between secondary and primary care.

Method All letters to GPs of patients over 18 with a Leeds postcode previously under the care of an oncologist and who died in January $2010(n=79)$ and January 2014 $(n=82)$ were included. Patients whose treatment was curative and more than 10 years before their death were excluded if they died of an unrelated cause.

The improvement plan implemented between the two audits involved an oncology consultant participating in the development programme working alongside a GP in an Action Learning Set and spending time with District Nurses to understand their roles. He then worked with colleagues to highlight the importance of communication with GPs, patients and carers.

Results There was a statistically significant improvement in all desired characteristics of the letters between 2010 and 2014. This was most marked in recommendation to add the patient to the palliative care register. There was also a major improvement in documenting discussions with patients about the palliative nature of the disease and emphasis on quality of life.

Conclusion This audit shows that educational initiatives with system change can alter consultants' behaviour. This was a sustained effect, as funding for the development programme finished 2 years before re-auditing. Whilst other factors may contribute, it does demonstrate that long term change in consultant behaviour is possible, particularly when initiated by their peers with insight into department specific issues.

\section{P-31 A RETROSPECTIVE AUDIT OF SURVIVAL DURATION IN PATIENTS WITH POOR PERFORMANCE STATUS RECEIVING SYSTEMIC ANTI-CANCER THERAPY AT MID YORKSHIRE NHS TRUST}

1,2Emma Hooson, ${ }^{2}$ Charlotte Gibb, ${ }^{2}$ Gireesh Kumaran. 'St Gemma's Hospice, Leeds, UK; ${ }^{2}$ Pinderfields Hospital, Mid Yorkshire Hospitals NHS Trust, Wakefield, UK

\subsection{6/bmjspcare-2017-00133.31}

Background Research undertaken in patients with a cancer diagnosis and poor performance status (PS) has shown a lack of survival benefit or improved quality of life from chemotherapy. ${ }^{1,2,3}$ A review by the National Confidential Enquiry into Patient Outcome and Death found that poor PS was linked to an increased rate of death within 30 days of systemic anti-cancer therapy (SACT). ${ }^{4}$ We analysed survival duration in patients with a poor PS prescribed SACT in our local NHS trust.

Methods Details for all patients who were prescribed SACT in the Mid Yorkshire Hospitals NHS Trust from 1/1/2015 to 31/ $12 / 2015$ were obtained from the electronic prescribing system (chemocare) and electronic case records (PPM). Patients included had an Oncological ICD-10 classification and had a performance status of 2 or less at the start of cycle 1 of treatment. Duration of survival for these patients was calculated.

Results From the total 747 patients, 39 were identified to fit the criteria. 33 had a PS of 2, and 6 had PS of 3 at the first cycle of SACT. Common diagnoses were lung carcinoma $(n=23)$ and breast carcinoma $(n=8) .35$ patients received chemotherapy with palliative intent. 3 of these patients died within 30 days of receiving SACT, and a total of 8 patients died within 60 days (23\%). All 4 patients who received neoadjuvant, adjuvant or disease modifying chemotherapy were still alive at time of analysis, with a survival of at least 421 days.

Conclusions Nearly 1 in 4 patients with a PS of 2 or less who were prescribed palliative chemotherapy had died within 60 days of receiving SACT. Quality of life and best supportive care need to be the first consideration for patients with poor PS, but carefully chosen and counselled patients with chemosensitive disease can benefit from SACT (further research needed).

\section{REFERENCES}

1. Sánchez-Muñoz, Pérez-Ruiz, Sáez Ml, et al. Limited impact of palliative chemotherapy on survival in advanced solid tumours in patients with poor performance status. Clin Transl Oncol 2011;13(6):426-9.

2. Wright $A A$, Zhang $B$, Keating $N L$, et al. Associations between palliative chemotherapy and adult cancer patients' end of life care and place of death: prospective cohort study. BMJ 2014:4:348.

3. Prigerson $\mathrm{H}$, Bao $\mathrm{Y}$, Shah $\mathrm{M}$, et al. Chemotherapy Use, Performance Status, and Quality of Life at the End of Life. JAMA Oncol 2015:1 (6):778-784.

4. Mort D, Lansdown M, Smith N, et al. Systemic Anti-Cancer Therapy: For better for worse? National Conï $\neg$ dential Enquiry into Patient Outcome and Death [Online] Available at: http://www.ncepod.org.uk/2008report3/Downloads/ SAC T_report.pdf (accessed September 4 th, 2016) 